\title{
A CHARACTERIZATION OF CERTAIN COMPACT FLOWS ${ }^{1}$
}

\author{
RONALD A. KNIGHT
}

\begin{abstract}
Compact flows on certain 2-manifolds are characterized in terms of the bilateral stability properties of the orbits.
\end{abstract}

1. Introduction. Beck and $\mathrm{Wu}$ have obtained topological characterizations of compact flows on certain surfaces [1], [7]. The major result of this paper is the characterization of such closed flows on certain 2-manifolds in terms of the flow structure itself. In particular, compact flows are characterized by the stability properties of the periodic orbits and critical points.

A pair $(X, \pi)$ consisting of a topological space $X$ and a continuous mapping $\pi: X \times R \rightarrow X$, from the product space $X \times R$ into $X$, where $R$ denotes the real numbers, is called a dynamical system or (continuous) flow whenever $\pi(x, 0)=x$ for each $x$ in $X, \pi(\pi(x, t), s)=\pi(x, t+s)$ for each $x$ in $X$ and $t$ and $s$ in $R$, and $\pi$ is continuous on $X \times R$. An orientable 2-manifold $X$ is said to be dichotomic if every simple closed curve $C$ in $X$ decomposes $X$ into two connected sets with common boundary $C$. The phase spaces considered in this paper are dichotomic.

We shall denote the trajectory (orbit) through $x$, orbit closure of $x$, limit set of $x$, and prolongation of $x$ by $C(x), K(x), L(x)$, and $D(x)$, respectively. For basic properties of dynamical systems we refer the reader to [2] and [3].

A flow is of characteristic 0 if and only if $D(x)=K(x)$ for each $x$ in the phase space. A flow is called compact or periodic provided each of its orbits is compact. Throughout the paper we shall use $P$ and $S$ to denote the sets of periodic and critical points of a given flow, respectively. Note that an orbit is compact if and only if it is in $P \cup S$.

The extension of a flow $(X, \pi)$ to the one point compactification $X^{*}$ of $X$ is denoted by $\left(X^{*}, \pi^{*}\right)$. For each function on $X$ we shall denote its extension to $X^{*}$ with the superscript ${ }^{*}$. For a given flow we use $S^{*}$ to denote the critical points of the extended flow.

\section{Preliminary results.}

LeMma 1. Let $(X, \pi)$ be a compact flow with a dichotomic phase space. If $S_{0}$ is a compact component of $S$, then $S_{0}$ is bilaterally stable.

Received by the editors August 13, 1976.

AMS (MOS) subject classifications (1970). Primary 34C34, 54H20; Secondary 34D30, 58F10.

Key words and phrases. Bilateral stability, characteristic 0, closed flow, compact flow, dichotomic space, dynamical system, flow, 2-manifold, stability.

${ }^{1}$ This research was partially supported by an NMSU faculty research grant. 
Proof. For each point $x$ in $X-S, C(x)$ is bilaterally stable according to the Cycle Stability Theorem [3, 3.3, p. 196] yielding $D(x)=D(C(x))=$ $C(x)\left[2,7.6\right.$, p. 77]. Thus, $D(X-S)=X-S$ and $D(S)=S$. For a point $s_{0}$ in $S_{0}$ the set $D\left(s_{0}\right) \cap S_{0}$ is compact and contains a compact component of $D\left(s_{0}\right)$. Thus, $D\left(s_{0}\right)=D\left(s_{0}\right) \cap S_{0}\left[2,6.12 .2\right.$, p. 68], and hence, $D\left(S_{0}\right)=S_{0}$. The proof is complete.

Proposition 2. Let $(X, \pi)$ be a compact flow and $X$ be dichotomic. If $S$ is totally disconnected, then $(X, \pi)$ is of characteristic 0 .

Proof. According to the Cycle Stability Theorem and Lemma 1, each trajectory is bilaterally stable. Hence, $D(x)=D(C(x))=C(x)=K(x)$ for each point $x$ in $X$.

The only planar compact flows of characteristic 0 are critical flows and flows consisting of a global Poincaré center [5].

Proposition 3. Let $(X, \pi)$ be a compact flow with dichotomic phase space and let $S$ be totally disconnected. Then (i) if $S$ is empty, $X$ is an open annular region; (ii) if $S$ is nonempty, one of the following holds:

(a) $X$ is homeomorphic to $R^{2}$ and $S$ consists of a global Poincaré center; or

(b) $X$ is homeomorphic to $S^{2}$ and $S$ consists of two Poincaré centers $s_{1}$ and $s_{2}$ such that $s_{i}$ is a global Poincaré center relative to $X-\left\{s_{j}\right\}, i \neq j$.

Proof. The torus, Mobius strip, and Klein bottle are not dichotomic spaces so that Proposition 6.2 of [7] applies, and hence, $X-S$ consists of the disjoint union of open annuli. If $S=\varnothing$, the connectedness of $X$ implies that $X$ consists of exactly one annular region. If $S \neq \varnothing, S$ consists of Poincaré centers since $(X, \pi)$ is of characteristic $0[4,10]$. Each point of $S$ is in the boundary of exactly one annular region. If $S$ consists of one Poincaré center, then the connectedness of $X$ implies that $X$ contains a single annular region, and hence, is homeomorphic to $R^{2}$. Suppose that $S$ consists of at least two points. Let $x$ be an element of $S$ and let $H$ be the annular region whose boundary contains $x$. If no point of $S-\{x\}$ is in $\bar{H}$, then $\bar{H}$ and $X-\bar{H}$ are disjoint closed sets. This is clearly impossible since $X$ is connected. Hence, there is a point $y$ in $S \cap \bar{H}$ distinct from $x$. The set $\bar{H}$ must be $H \cup\{x, y\}$ and $\bar{H}$ is homeomorphic to $S^{2}$. If $S \neq\{x, y\}$, then $\bar{H}$ and $X-\bar{H}$ are disjoint closed sets which is absurd. Hence, $X=\bar{H}$ and $X$ is homeomorphic to $R^{2}$.

3. Characterization. Seibert and Tulley showed [6] that a planar flow is compact if and only if it is Poisson stable at each point. Hajek extended the result to dichotomic phase spaces [3, 1.6, p. 183]. According to Beck [1] the set of periodic points of a planar compact flow is composed of countably many open periodic annular regions. He thus characterized such flows in terms of the complement of their critical points. Wu obtained similar results [7] for certain 2-manifolds. In the following theorem we characterize compact flows on a dichotomic phase space in terms of the bilateral stability of $S^{*}$ and the orbits in $P$. 
THEOREM 4. A flow on a dichotomic phase space $X$ is compact if and only if each component of the boundary of $S^{*}$ and each periodic orbit are bilaterally stable in $X^{*}$.

Proof. Throughout the proof we shall refer to the topology on $X^{*}$. Let $(X$, $\pi$ ) be compact and let $K$ be a component of the boundary of $S^{*}$. The component $S_{0}$ of $S$ which contains $K$ is bilaterally stable by Lemma 1 , and hence, $\delta S_{0}$ and $K$ are bilaterally stable [3, 4.13.4, p. 114]. Furthermore, each periodic orbit is bilaterally stable by the Cycle Stability Theorem.

Conversely, we first show that $P$ is open. Let $C(x)$ be any periodic orbit. Since $C(x)$ is bilaterally stable and $S^{*}$ is closed there is a compact neighborhood $V$ of $C(x)$ disjoint from $S^{*}$. Let $y$ be any point of $V$. Then either $L^{*}(y) \subset P$ or, for each $z \in L^{*}(y)$, we have $L^{*}(z) \subset S^{*}[3,1.11$, p. 184]. Since $V \cap S^{*}=\varnothing$, we have $L^{*}(y) \subset P$. Suppose $y$ is not periodic and let $z$ be any point of $L^{*}(y)$. Then $y \notin L^{*}(y)$ and there is a compact invariant neighborhood $U$ of the periodic orbit $C(z)$ excluding $y$. In fact, there is a compact invariant neighborhood $W$ of $C(z)$ contained in the interior of $U$. But this means that $L^{*}(y) \subset X^{*}-W$, and hence, that $z \notin L^{*}(y)$, which is absurd. Thus, $y \in L^{*}(y)$ which means that $y$ is periodic and $C(x) \subset V \subset P$. Hence, $P$ is open.

Next, we let $x \in X^{*}-P \cup S^{*}$. Then either $L^{*}(x) \subset P$ or $L^{*}(y) \subset S^{*}$ for each $y$ in $L^{*}(x)$. Since $P$ is open, $L^{*}(x) \not \subset P$. The bilateral stability of each component of $\delta S^{*}$ implies that $\delta S^{*}$, and hence, $S^{*}$ are bilaterally stable. There is an open invariant neighborhood $V$ of $S^{*}$ whose closure excludes $x$. Thus, $L^{*}(x) \subset X^{*}-V$ so that $L^{*}(y) \subset X^{*}-V$ for each $y$ in $L^{*}(x)$ which means, of course, that $L^{*}(y) \not S^{*}$ for any $y$ in $L^{*}(x)$. This contradiction yields $X^{*}=P \cup S^{*}$.

Corollary 4.1. A flow on a dichotomic space $X$ is compact if and only if $S^{*}$ and each periodic orbit are bilaterally stable in $X^{*}$.

COROLlARY 4.2. The only compact flows on a dichotomic phase space having each orbit bilaterally stable are those where $X=P, X=S, S$ consists of a global center, or $X=S^{2}$ and $S$ consists of two centers.

\section{REFERENCES}

1. A. Beck, Plane flows with closed orbits, Trans. Amer. Math. Soc. 114 (1965), 539-551. MR 30 \# 5290.

2. N. Bhatia and O. Hájek, Local semi-dynamical systems, Lecture Notes in Math., Vol. 90 , Springer-Verlag, Berlin and New York, 1969. MR 40 \#4559.

3. O. Hajek, Dynamical systems in the plane, Academic Press, New York, 1968. MR 39 \# 1767.

4. R. Knight, Certain closed flows on a 2-manifold (manuscript).

5. __ Dynamical systems of characteristic 0, Pacific J. Math. 41 (1972), 447-457. MR 47 \#2578.

6. P. Seibert and P. Tulley, On dynamical systems in the plane, Arch. Math. (Basel) 18 (1967), 290-292. MR 36 \#873.

7. T. Wu, Continuous flows with closed orbits, Duke Math. J. 31 (1964), 463-469. MR 29 \# 1631.

Mathematics Division, Northeast Missouri State University, Kirksville, Missouri 63501 